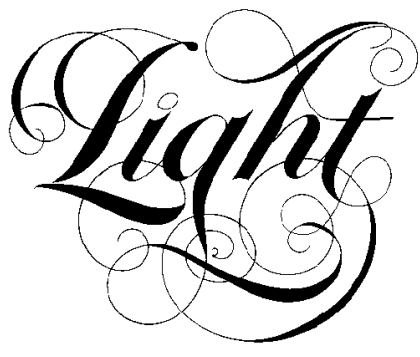

\title{
Effects of High Temperature and Pressure on Silica Optical Fibre Sensors
}

\author{
J. R.Clowes , J.McInnes ${ }^{(b)}$, M.N.Zervas, D.N.Payne
}

Sensor Dynamics, Abbas Business Centre, Itchen Abbas, Winchester, Hampshire, UK.

\begin{abstract}
We report on the effects of liquids at high temperature and pressure on silica optical fibres, sensors and gratings. We propose that the diffusion of molecules into the silica and the resultant expansion of the network are responsible for observed fibre expansions of up to $0.2 \%$ and Bragg wavelength increases of $2 \mathrm{~nm}$ at $1525 \mathrm{~nm}$. Amorphous carbon hermetic coating has shown a reduction of these effects by an order of magnitude at $300^{\circ} \mathrm{C}$. These results have strong implications for the deployment of fibre sensors in oil wells.
\end{abstract}

\section{Introduction:}

Many optical fibre sensors rely on the fact that the measurand modulates the optical path length and/or refractive index of the "stable" silica waveguide. If the long-term stability of silica is questionable, the accuracy of such sensors is dubious.

In this paper, we present recent results which demonstrate some of the problems of silica optical fibres as sensors in high temperature and pressure environments and report on the need for suitable "hermetic" coatings for such devices.

By monitoring the pressure-induced birefringence of a side-hole optical fibre [1] under conditions of high temperature and pressure, we have found that the birefringence drifts considerably. This drift increases non-linearly with increasing temperature and the effect is permanent.

The high temperature instabilities have been further investigated using $\mathrm{Hi}-\mathrm{Bi}$ fibre pressure sensors, fibre Bragg gratings and standard telecommunications single-mode fibre. Similar results for the drift in all cases show that the observed effects are inherent to silica and are not due to geometry. It is therefore expected that similar effects will be observed for any silica interferometric or polarimetric sensor in high temperature fluid environments.

\section{Side-Hole Polarimetric Fibre Sensor}

Side-hole fibre was proposed by Xie et al [1] as a possible sensor for hydrostatic or acoustic pressure. In the presence of hydrostatic pressure acting on the outside of the fibre, an anisotropic stress is induced in its core due to the fibre geometry. The resulting birefrigence is therefore a measure of the applied pressure.

The pressure sensor consists of an input polariser, a length of side-hole fibre and a mirror. The input polariser is aligned at $45^{\circ}$ to the birefringent axes of the side-hole fibre to couple approximately equal amounts of light into each axis. Under the application of hydrostatic pressure, a change in the refractive index in each axis is produced through the photoelastic effect. The result is the generation of a fast and slow axis, where $n_{f}$ and $n_{s}$ are the fast and slow indices 
respectively. The magnitude of $\Delta \mathrm{n}$, where $\Delta \mathrm{n}=\mathrm{n}_{\mathrm{S}}-\mathrm{n}_{\mathrm{f}}$, is dependent upon the applied pressure and also upon the fibre geometry.

The pressure-induced optical path difference between the birefringent fibre axes is monitored using a dynamically-matched, low-coherence interferometer in which the path difference is matched in a reference Hi-Bi fibre scanned in temperature. Measurements are made at $1300 \mathrm{~nm}$.

Hydrostatic pressure is applied to the fibre by means of a dead-weight tester through a liquid medium within high-pressure steel tubing. The chamber temperature is varied as required using heating cord.

Figure 1 shows the results of two $0.15 \mathrm{~m}$ long sensors at temperatures of 200 and $300^{\circ} \mathrm{C}$, where water has been used as the pressure medium. A pressure of 4000 psi was applied to the fibres. The resulting birefringence and corresponding pressure measurement are shown. The effect of temperature on the drift is very noticeable and increasing temperature has a disproportionate effect on the drift rate. The drift is therefore not observed at room temperature as the rate of change in birefringence is extremely low. For fibres at $300^{\circ} \mathrm{C}$, however, there is a zero crossing on the birefringence/pressure axis indicating that there has been an exchange of the birefringent fibre axes with the fast axis becoming slow and vice versa. This large drift effect is permanent and therefore, as the initial zero pressure offset of the fibre is low, on removal of the pressure, the resulting side-hole fibre is very highly birefringent (equivalent to a pressure of 6000psi) with a typical beat length of the order of $1.5 \mathrm{~mm}$ at $633 \mathrm{~nm}$. It was found that this can be reduced through HF etching of the fibre or by heat treating in an oven at high temperature.

As well as the temperature effect on the fibre drift, the effect of the liquid medium has also been studied. Figure 2 shows some of these results, all at approximately $300^{\circ} \mathrm{C}$. Other liquids have been used in an attempt to remove all trace amounts of water from the pressure chamber. It is believed that liquid ingress into the outer layers of the fibre cladding results in the development of a highly stressed layer which exerts an opposing "pressure" on the core. This has been confirmed through the use of a finite element model (FEM) of the fibre in which the introduction of a swollen surface layer is capable of inducing such a highly birefrefringent fibre. It is interesting to notice that the water has by far the greatest effect on the fibre and, for this reason, water has been used for the majority of recent experiments.

\section{Effect on Silica Fibres}

In order to investigate the effect of the fibre side-holes on the drift phenomenon, the pressure sensor experiment has been repeated using Bow Tie and Panda Hi-Bi fibres as pressure sensors. The resulting permanent-induced birefringence, normalised to pressure, was consistent with that for side-hole fibre, indicating that the fibre geometry and composition do not make a significant contribution to the drift rate.

To investigate this further, measurements have been made using single-mode fibre and negativeindex fibre Bragg gratings (to prevent high temperature decay [2]). The postulated swelling of a surface layer on the fibre is anticipated to produce an overall lengthening of the fibre. The length of a fibre and also the Bragg wavelength of a grating have, therefore, been monitored under similar conditions to the side-hole fibre. Lengths of carbon-coated fibre have also been measured as a comparison to uncoated fibre and as a test of the coating hermeticity. 
A length of carbon-coated fibre, a length of standard telecommunications fibre and a negative index grating were spliced to high-pressure seals in both a heated air chamber and a chamber containing water. The lengths were measured using low-coherence interferometry and the gratings were interrogated using an optical spectrum analyser and subsequent averaging of the spectrum to achieve a single Bragg wavelength value. The lengths of fibre within the pressure chamber were all $1.8 \mathrm{~m}$ with mirrored endfaces. However, only approximately $0.6 \mathrm{~m}$ of the fibre was in the hot area of the chamber.

Figure 3 shows the results for the length change of the fibre with time and Figure 4 is that for the change in Bragg wavelength with time. Both curves indicate that the same process is occurring for the two fibres. The sudden increase in fibre length and Bragg wavelength after 7 days occurred when the temperature of the chamber was lowered to $200^{\circ} \mathrm{C}$ and then returned to $300^{\circ} \mathrm{C}$, a procedure followed because of the fading of the grating reflection. However, after the temperature cycle had been made, the full grating reflection had returned and the fibre length and Bragg wavelength experienced sudden increases. This occurrence is being investigated further. These effects were not noticed in the carbon-coated fibre or the fibres in the dry chamber.

On removal of the fibres from the pressure chamber after the experiment, the lengths of fibre were physically measured and were observed to have increased in length more than the optical path length measurement of $1.1 \mathrm{~mm}$ suggested. The difference between the length change and opticalpath-length change indicates that there has been a decrease in the fibre index during the process. This effect is to be expected when a swollen cladding layer exerts a tensile stress on the fibre core.

The curve for the carbon-coated fibres in Figure 3 demonstrates a solution to the problem of liquid ingress into optical fibres, since it shows a reduction of the effect by a factor of approximately 10 .

\section{Theory of Drift Process}

We postulate that the drift phenomenon in optical fibres at high temperatures and pressure is due to the ingress of liquid molecules into the silica structure and the resultant disruption of the silica network. This disruption gives rise to an expansion of the glass and hence results in a tensile stress on the fibre core. In the case of the side-hole pressure sensor, this is shown as a change in birefringence of the fibre due to the fibre geometry. For lengths of fibre and fibre Bragg gratings, the result is the combination of a longitudinal strain and a decrease in the refractive index of the waveguide, which has the effect of masking the true magnitude of the fibre extension.

Modelling of this process using FEM shows that all the observed changes in the fibres can be explained by the swelling of a thin outer layer of the fibre. Also, etching experiments on the treated grating and side-hole fibres showed that the changes can be reversed. Figure 5 shows the results of etching the two Bragg gratings in a dilute solution of HF down to a radius of 


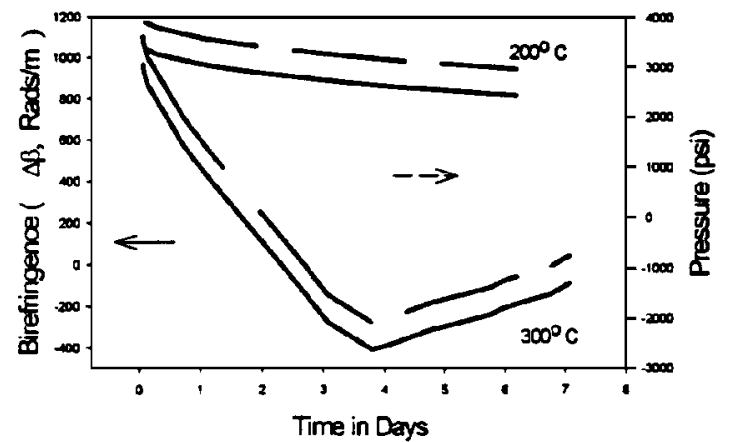

Fig.1: Side hole fibre birefringence (solid lines) and pressure drift (dashed lines) with time at $200^{\circ} \mathrm{C}$ and at $300^{\circ} \mathrm{C}$ in water under $4000 \mathrm{psi}$.

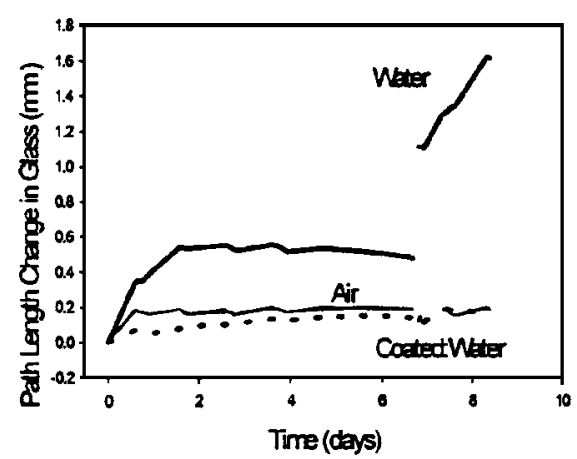

Fig.3: Length change of coated (dashed lines) and uncoated fibres (solid) in water and air at $300^{\circ} \mathrm{C}$ with time.

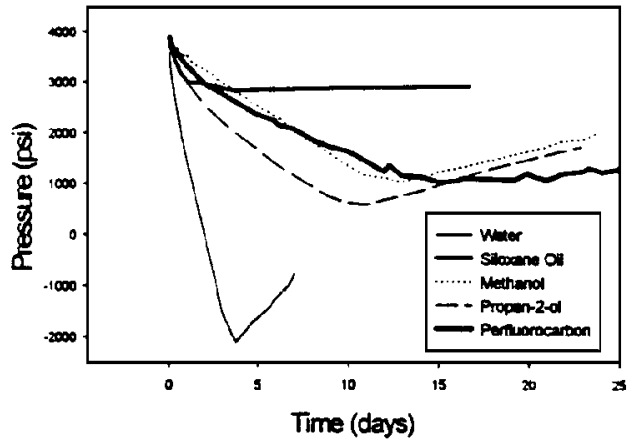

Fig.2: Side hole fibre pressure drift with time at $300^{\circ} \mathrm{C}$ in various liquids under $4000 \mathrm{psi}$.

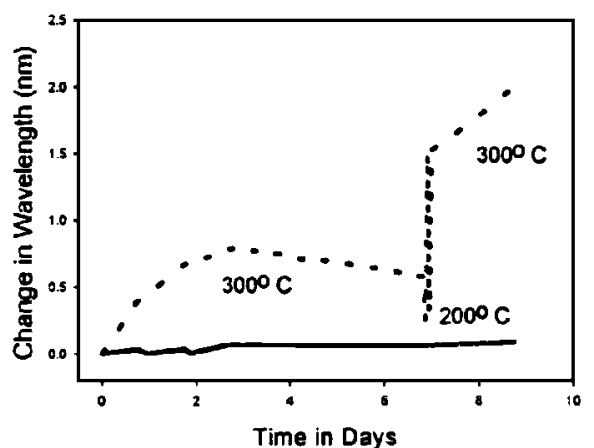

Fig.4:Change in bragg wavelength of fibre bragg gratings in water (dashed) and air at $300^{\circ} \mathrm{C}$ under 4000 psi.

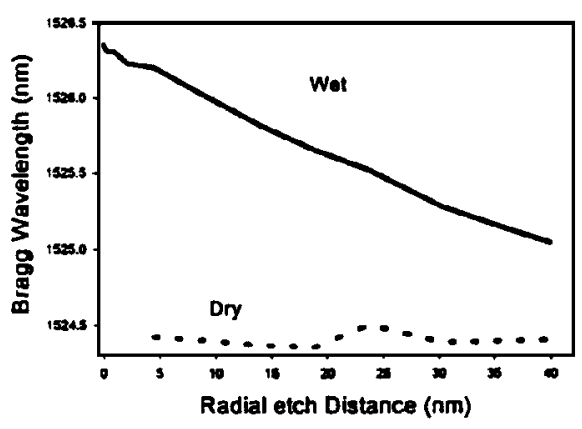

Fig.5: Change in bragg wavelength of water treated and dry treates (dashed) gratings during HF Etching. 
approximately $15 \mu \mathrm{m}$. In untreated fibres little change in Bragg wavelength is expected down to this fibre diameter [3]. Similar behaviour was observed with the treated "dry" grating. However, the Bragg wavelength of the "wet" grating has shifted considerably from its offset value of $1526.39 \mathrm{~nm}$ back towards its initial (untreated) wavelength of $1524.49 \mathrm{~nm}$. The observed change continued to occur until the end of the etch experiment when the fibres failed at a measured

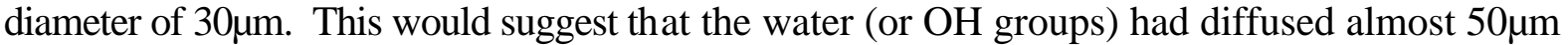
into the glass, which is much greater than has been previously shown in bulk glasses during autoclave experiments [4] where a value for the diffusion coefficient of approximately $5 \times 10^{-}$ ${ }^{13} \mathrm{~cm}^{2} / \mathrm{s}$ has been given for $300^{\circ} \mathrm{C}$. From this diffusion coefficient, the average diffusion distance of a water molecule (or hydroxyl group) would be approximately $8 \mu \mathrm{m}$.

\section{Conclusions}

We have shown results which question the stability of silica fibres in high temperature and pressure conditions and have attributed this instability to the diffusion of the surrounding medium constituents into the glass. This has great implications for the use any optical fibre sensors, especially in harsh conditions such as down-hole sensing in oil wells. Long-term drifts at lower temperatures are also therefore inevitable, althought the effects are considerably less at reduced temperatures. Finally, we present a solution to this problem with the use of hermetic coatings, in this case carbon, which has reduced the effects by an order of magnitude at $300^{\circ} \mathrm{C}$.

We acknowledge useful discussions with Dr. M. Varnham and Dr. E. Kluth. We would like to thank Sensor Dynamics and Chevron for sponsorship of this work and also Dr. K. Walker of Lucent Technologies for the donation of carbon-coated hermetic fibre.

\section{References}

1. H.M.Xie et al, "Side-hole fiber for fiber-optic pressure sensing", Optics Letters, Vol. 11, No.5, 1986.

2. W.F.Liu et al, "Comparison of the Decay Behaviour of Negative and Positive Index Change Fibre Bragg Gratings", IEE Colloq. Optical Fibre Gratings, London, 7/2/1997.

3. L.Dong et al, "Tuning and Chirping Fiber Bragg Gratings by Deep Etching”, IEEE Photonics Technology Letters, Vol. 7, No.12, 1995.

4. H.Wakabayashi and M.Tomozawa, "Diffusion of Water into Silica Glass at Low Temperature”, J. Am. Ceram. Soc, Vol. 72, No.10, pp. 1850-55, 1989. 